\title{
BMJ Global Health Planning prompts to promote uptake of HIV services among men: a randomised trial in rural Uganda
}

\author{
Nolan M Kavanagh (D) , ${ }^{1}$ Elisabeth M Schaffer, ${ }^{2}$ Alex Ndyabakira, ${ }^{3}$ Kara Marson, ${ }^{4}$ \\ Diane V Havlir, ${ }^{4}$ Moses R Kamya, ${ }^{3,5}$ Dalsone Kwarisiima, ${ }^{3}$ Gabriel Chamie, ${ }^{4}$ \\ Harsha Thirumurthy ${ }^{1,6}$
}

To cite: Kavanagh NM,

Schaffer EM, Ndyabakira A, et al Planning prompts to promote uptake of HIV services among men: a randomised trial in rural Uganda. BMJ Global Health 2020;5:e003390. doi:10.1136/ bmjgh-2020-003390

Handling editor Valery Ridde

- Additional material is published online only. To view, please visit the journal online (http://dx.doi.org/10.1136/ bmjgh-2020-003390).

Received 8 July 2020 Revised 20 0ctober 2020 Accepted 25 October 2020

\section{Check for updates}

(c) Author(s) (or their employer(s)) 2020. Re-use permitted under CC BY-NC. No commercial re-use. See rights and permissions. Published by BMJ.

For numbered affiliations see end of article.

\section{Correspondence to} Dr Harsha Thirumurthy; hthirumu@pennmedicine. upenn.edu

\section{ABSTRACT}

Introduction Interventions informed by behavioural economics, such as planning prompts, have the potential to increase HIV testing at minimal or no cost. Planning prompts have not been previously evaluated for HIV testing uptake. We conducted a randomised clinical trial to evaluate the effectiveness of low-cost planning prompts to promote HIV testing among men.

Methods We randomised adult men in rural Ugandan parishes to receive a calendar planning prompt that gave them the opportunity to make a plan to get tested for HIV at health campaigns held in their communities. Participants received either a calendar showing the dates when the community health campaign would be held (control group) or a calendar showing the dates and prompting them to select a date and time when they planned to attend (planning prompt group). Participants were not required to select a date and time or to share their selection with study staff. The primary outcome was HIV testing uptake at the community health campaign

Results Among 2362 participants, 1796 (76\%) participants tested for HIV. Men who received a planning prompt were 2.2 percentage points more likely to test than the control group, although the difference was not statistically significant $(77.1 \%$ vs $74.9 \% ; 95 \% \mathrm{Cl}-1.2$ to 5.7 percentage points, $p=0.20$ ). The planning prompt was more effective among men enrolled $\leq 40$ days before the campaigns (3.6 percentage-point increase in testing; $95 \% \mathrm{Cl}-2.9$ to $10.1, p=0.27$ ) than among men enrolled $>40$ days before the campaigns (1.8 percentage-point increase; $95 \% \mathrm{Cl}-2.3$ to $5.8, \mathrm{p}=0.39$ ), although the effects within the subgroups were not significant.

Conclusion These findings suggest that planning prompts may be an effective behavioural intervention to promote HIV testing at minimal or no cost. Large-scale studies should further assess the impact and cost-effectiveness of such interventions.

\section{INTRODUCTION}

Increasing the uptake of HIV testing services among men is essential for meeting the UNAIDS 95-95-95 targets by 2030 and ending the AIDS epidemic in sub-Saharan Africa. Despite the scale-up of testing services, about

\section{Key questions}

What is already known?

- One-fifth of men living with HIV in sub-Saharan Africa remain unaware of their HIV status, and brief community health campaigns can increase HIV testing uptake rapidly and at low cost.

- 'Planning prompts', or implementation intentions, are an example of behavioural economics 'nudges' that can promote health behaviours by prompting people to voluntarily select a specific date and time when they will engage in the behaviour.

What are the new findings?

- We conducted a randomised clinical trial with 2362 adult men in rural parishes of Uganda to evaluate the effectiveness of planning prompts for the uptake of HIV testing.

- Men who received a calendar prompting them to select a date and time to visit a community health campaign were 2.2 percentage points more likely to get HIV testing, although the finding was not statistically significant.

- The planning prompt appeared to be more effective at encouraging HIV testing when it was delivered to men closer to the testing dates.

What do the new findings imply?

- Planning prompts may promote HIV testing at minimal or no cost, especially when delivered closer in time to opportunities for HIV testing.

- Larger studies should evaluate their impact and cost-effectiveness as well as other behavioural economics 'nudges' for HIV testing and care.

one-fifth of the men living with HIV in subSaharan Africa remain unaware of their status. ${ }^{2}$ Brief community health campaigns that offer testing have shown promise as a lowcost way to rapidly increase testing coverage. Since men are less likely to participate in these campaigns and seek HIV services more generally, ${ }^{23}$ behavioural interventions could promote men's uptake of such communitybased HIV testing services. 
Behavioural economics insights have been used to develop inexpensive 'nudges' and other interventions to promote a wide variety of behaviours, including organ donation, ${ }^{4}$ immunisation, ${ }^{5}{ }^{6}$ medication adherence ${ }^{78}$ and HIV testing. ${ }^{9}{ }^{10}$ Successful approaches include the provision of small incentives, use of defaults and deployment of reminders. These interventions encourage participants to undertake a behaviour by changing the conditions under which they make that choice while preserving their autonomy to decline. ${ }^{11}$ One relevant insight from behavioural economics is that people are more likely to engage in a desired behaviour in the near future when they develop an action plan for doing so. ${ }^{12}$ Such 'implementation intentions' or 'planning prompts' can be as simple as writing down a date and time when one will undertake the behaviour. They have been shown to generate small but meaningful increases in influenza vaccination rates in the $\mathrm{USA}^{5}$ and cervical cancer screening in the $\mathrm{UK}^{13}{ }^{13}$ decreases in smoking uptake among adolescents in the $\mathrm{UK}^{14}$ and effects on a variety of other health behaviours in various settings ${ }^{1516}$ at no additional cost.

We conducted a randomised trial to test whether planning prompts increased men's uptake of HIV testing at community health campaigns held in rural Uganda.

\section{METHODS}

This study was nested within another randomised trial (NCT02890459) of financial incentives to promote HIV testing that has been previously published. ${ }^{9}$ Between April and June 2016, we enrolled men aged $\geq 18$ years from villages in four rural parishes in Mbarara District, Uganda. After consenting, participants responded to a questionnaire and were randomised to one of six incentive arms (gain-framed, loss-framed and lottery rewards, each worth US $\$ 1$ or US\$5) for undergoing free HIV testing at multidisease community health campaigns (CHCs). Randomisation was done by blocks (block size $=30)$ with computer-generated allocation sequences before the initiation of trial enrolment by study investigators. The CHCs were to be held in participants' communities over 2 weeks in June-July 2016. A power calculation for the parent trial was anchored at 3000 participants given budget and population size constraints in the communities; based on this sample, the parent trial had $80 \%$ power to detect a 6 percentage-point difference in HIV testing between the gain-framed group and either other incentive group $(\alpha=0.05$, two-sided $) .{ }^{9}$ In the context of an incentive-based intervention, this effect size was considered meaningfully large for scale-up. Even so, there were not enough eligible men in the parishes that met enrolment criteria for 3000 participants. For the planning prompt substudy, a two-arm trial with the final participant count had $80 \%$ power to detect a difference in HIV testing of at least 5 percentage points, which would be considered a large effect size for a nudge intervention. ${ }^{17}$

Within each arm, participants were randomised (1:1) to a control group that received a calendar showing the days of the CHC or to a 'planning prompt' group that received the same calendar along with a suggestion that they circle the specific date and time (morning or afternoon) when they would come for an HIV test (figures 1 and 2). Participants with the planning prompt were told, 'many people find it helpful to make a plan for when to go and get tested', but were not required to make a selection

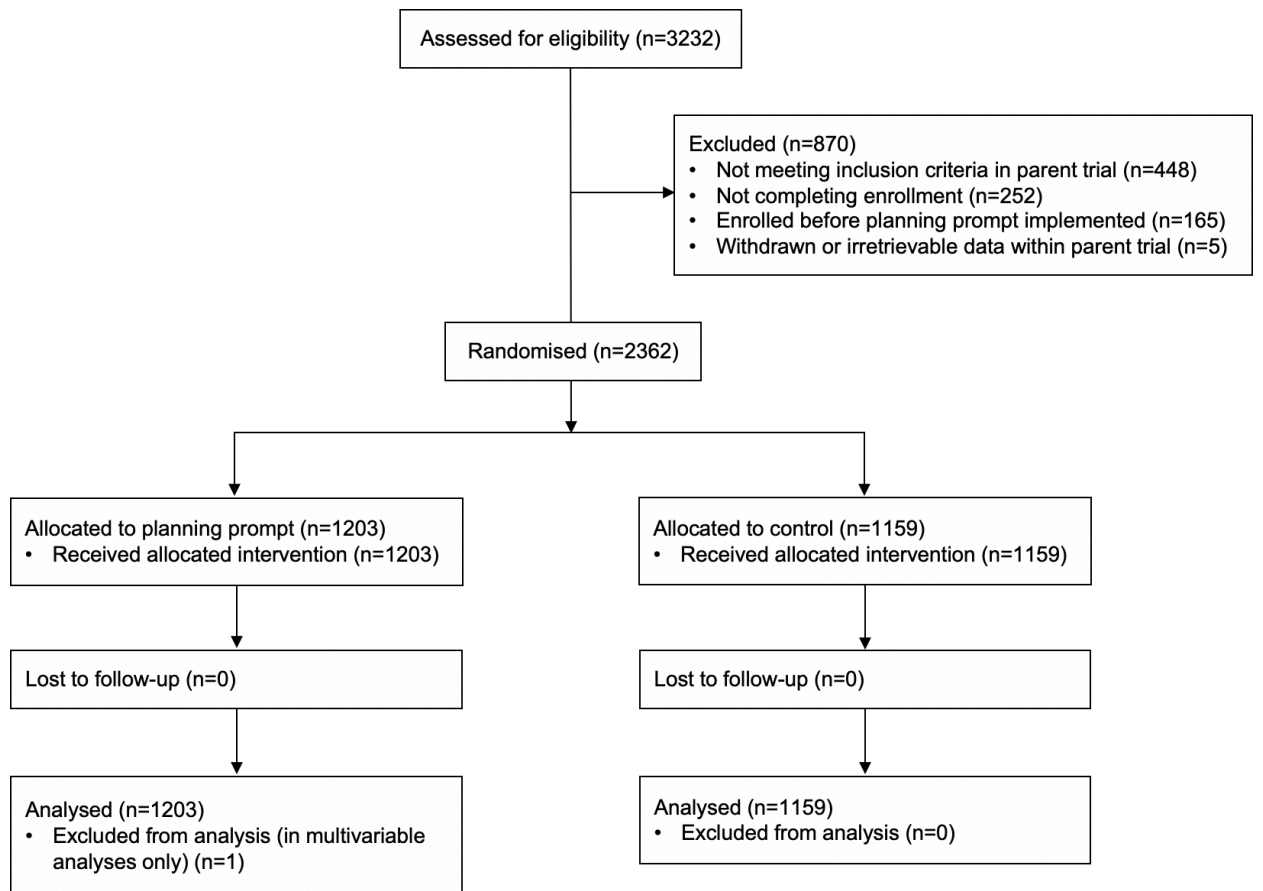

Figure 1 Consolidated Standards of Reporting Trials flow diagram. 


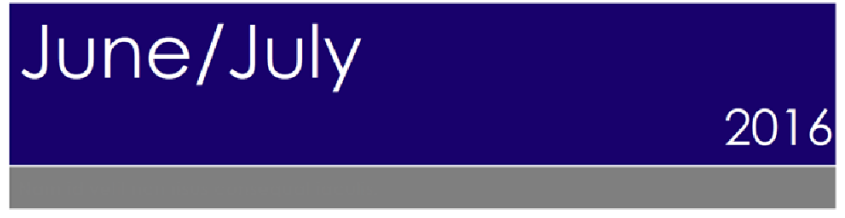

Sharamu kare!

Ehururu y'ebya amagara neyija omukyanga kyaawe amukwa mukaaga hamwe nokwamushanju. Ahansi, nebiro by'okwezi hamwe ne myanya eyl ehururu yebyamagara erabemu. Abantu bingi nikibayamba kweteb ekenisa bakasharamu obu beragyende ba kekyebeza. Mowenu nil we sharamu obwe rikutorona orunaku hamwe neshagha.

Plan ahead!

the community hea th campaign $(\mathrm{CHC})$ is coming to your community in June and July. Below are the dates and location of the CHC. Many people find it helpful to make a plan for when to go and get tested. Make your own plan by selecting o day and time below:

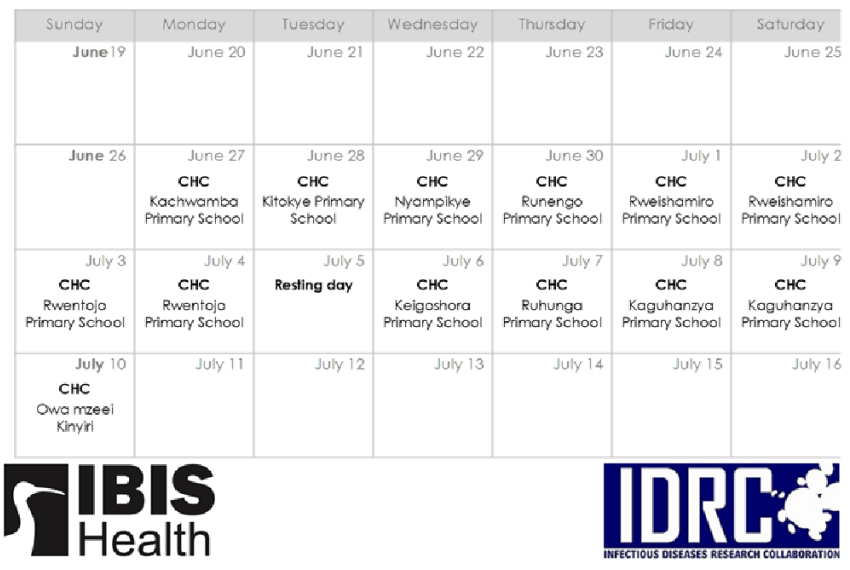

Planning prompt intervention group

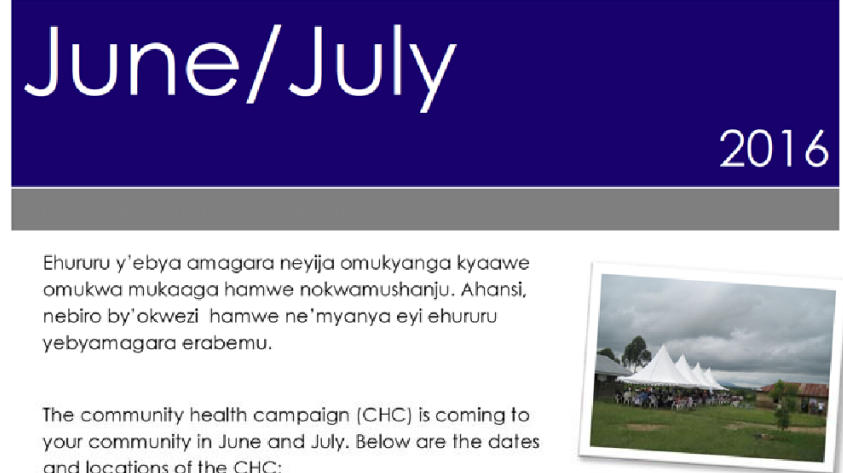
and locations of the $\mathrm{CHC}$ :

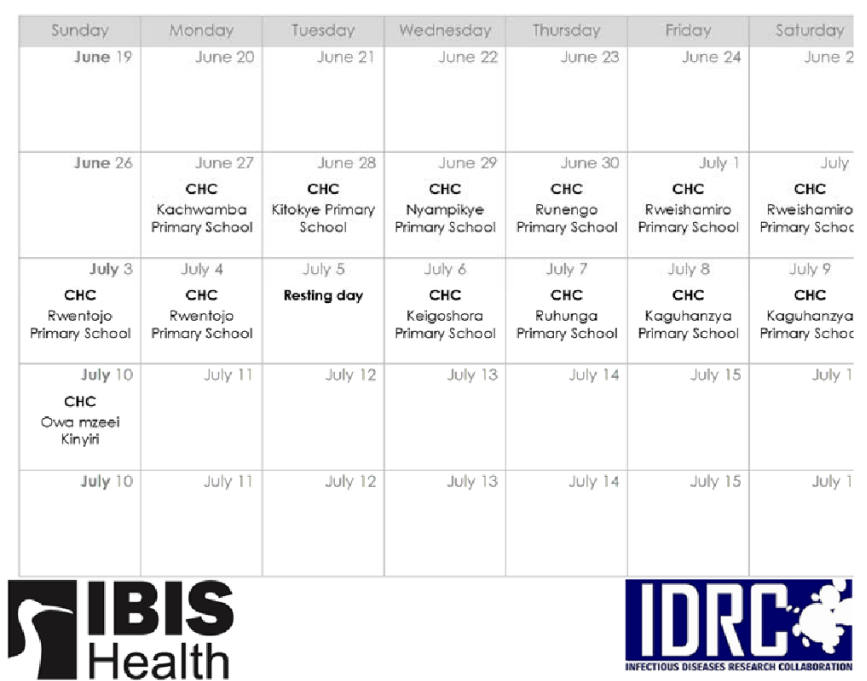

Control group

Figure 2 Calendars of community health campaign dates with and without planning prompt.

or show research assistants their selection. At the CHCs, participants received rapid HIV antibody testing by fingerprick blood collection in line with Ugandan Ministry of Health guidelines. ${ }^{18}$ The primary outcome of the trial was completion of HIV testing at a CHC, which was verified by fingerprint biometric measurement during both enrolment and at the CHC.

We used ordinary least squares (OLS) regression models to estimate the percentage-point change in the uptake of HIV testing due to the planning prompt. Additional multivariable models adjusted for incentive group, prize value, the interaction between incentive group and prize value, age, highest education level completed (primary or less, secondary, or more than secondary), and marital status (married/cohabitating, single, or separated/divorced/ widowed). While using OLS models for binary outcomes is common in economics research, ${ }^{19}$ sensitivity analyses using logistic regression models were also performed and are included in the online supplemental appendix. Since previous tests of planning prompts have shown benefits when the desired action is to be taken in the near future, ${ }^{5}$ in post hoc subgroup analyses we compared the effects of the intervention among participants enrolled $\leq 40$ days before the CHC and those enrolled 41-80 days before the CHC; 40 days was approximately half the range of days between enrolment and CHCs. Other post hoc subgroup analyses (by incentive arm and by testing for HIV in the past 12 months or not) were also performed and are included in the online supplemental appendix. We used $\alpha=0.05$ (two-sided) as the level for statistical significance. All analyses were performed in R (V.3.6.3).

\section{Patient and public involvement}

Community members participated early and frequently in the design and implementation of the trial. A community advisory board (CAB) was convened prior to study initiation and its members were consulted on the study design, development of testing campaigns and interventions to promote testing. Research assistants were introduced to community leaders and had support from them during enrolment and other study activities. Health services provided as part of the study were available to all community members. Study progress and results were shared with $\mathrm{CAB}$ members.

\section{RESULTS}

We enrolled 2532 men from rural Ugandan villages in the parent trial and randomised $2362(85 \%)$ of them to either the planning prompt group $(n=1203)$ or the control group $(\mathrm{n}=1159)$ (figure 1$)$. Not all participants in the parent trial were randomised because the planning 
Table 1 Participant characteristics by randomisation to a planning prompt for HIV testing

\begin{tabular}{|c|c|c|}
\hline & $\begin{array}{l}\text { Control group } \\
\text { No. }(\%)^{*}\end{array}$ & $\begin{array}{l}\text { Planning prompt } \\
\text { No. }(\%)^{*}\end{array}$ \\
\hline No. of participants enrolled & 1159 & 1203 \\
\hline Age (mean (SD)) & $38.7(15.9)$ & $38.5(15.8)$ \\
\hline \multicolumn{3}{|l|}{ Education } \\
\hline Primary or less & $859(74)$ & $890(74)$ \\
\hline Secondary & $207(18)$ & $233(19)$ \\
\hline More than secondary & $93(8)$ & $79(7)$ \\
\hline \multicolumn{3}{|l|}{ Marital status } \\
\hline Married/Cohabitating & $787(68)$ & $804(67)$ \\
\hline Single & $297(26)$ & $321(27)$ \\
\hline Separated/Divorced/Widowed & $75(6)$ & $78(6)$ \\
\hline \multicolumn{3}{|l|}{ Daily wage (US\$)† } \\
\hline Mean (SD) & $2.04(5.32)$ & $1.76(3.51)$ \\
\hline Median (IQR) & $1.05(0.60-1.79)$ & $0.90(0.60-1.79)$ \\
\hline \multicolumn{3}{|l|}{ Parish } \\
\hline Mabira & $371(32)$ & $361(30)$ \\
\hline Ruhunga & $324(28)$ & $357(30)$ \\
\hline Katyazo & $392(34)$ & $410(34)$ \\
\hline Itara & $72(6)$ & $74(6)$ \\
\hline Has ever had an HIV test & $899(78)$ & $933(78)$ \\
\hline HIV test in the past 12 months & $459(40)$ & $448(37)$ \\
\hline \multicolumn{3}{|l|}{ Perceived risk of HIV } \\
\hline No risk at all & $374(32)$ & $384(32)$ \\
\hline Low risk & $522(45)$ & $524(44)$ \\
\hline Moderate to high risk & $236(20)$ & $267(22)$ \\
\hline Unknown risk & $26(2)$ & $27(2)$ \\
\hline \multicolumn{3}{|c|}{ Likelihood of testing for HIV at a community health event in the future } \\
\hline Very likely & 1101 (95) & $1132(94)$ \\
\hline Somewhat likely & $45(4)$ & $55(5)$ \\
\hline Very unlikely & $7(1)$ & $5(0)$ \\
\hline Do not know & $2(0)$ & $2(0)$ \\
\hline
\end{tabular}

${ }^{*}$ Data are no. (\%), unless otherwise specified.

†Average exchange rate during enrolment period: 3344 UGX=1 US\$.

prompt was implemented beginning on the fourth day of enrolment. All participants were analysed by their assigned groups, and all except one (>99\%) had full data for the adjusted analyses. Participants' characteristics were similar between the two study groups (table 1). Their mean age was 38.6 years $(\mathrm{SD}=15.9), 74 \%$ reported their highest level of completed education as primary or less and $67 \%$ were married or cohabitating. Seventy-eight per cent of participants reported having ever been tested for HIV and $38 \%$ had been tested within the past year.

Overall, $1796(76 \%)$ participants tested for HIV at a CHC. Participants in the planning prompt group were 2.2 percentage points more likely to test at the $\mathrm{CHC}$ than the control group, although the difference was not statistically significant $(77.1 \%$ vs $74.9 \%$; $95 \%$ CI -1.2 to
5.7 percentage points, $\mathrm{p}=0.20$ ) (table 2 ). Adjusting for the incentive group to which participants were assigned in the parent trial and for key demographic characteristics (age, education and marital status), the effect of the planning prompts on HIV testing increased to 2.5 percentage points while remaining statistically insignificant $(95 \% \mathrm{CI}$ -0.9 to $5.9, \mathrm{p}=0.15$ ). The main adjusted model is provided in full in the appendix (online supplemental appendix table 1). Results were similar when analysed with logistic regression models (online supplemental appendix table 2).

Among 1775 participants who were enrolled $>40$ days before the CHC, the planning prompt increased HIV testing uptake by 1.8 percentage points $(95 \% \mathrm{CI}-2.3$ to 5.8, $\mathrm{p}=0.39$ ) (online supplemental appendix table 3). By 
Table 2 HIV testing at community health campaigns due to planning prompts

\begin{tabular}{|c|c|c|}
\hline & Control group & Planning prompt \\
\hline No. of participants & 1159 & 1203 \\
\hline \multicolumn{3}{|l|}{ HIV testing uptake } \\
\hline $\mathrm{N}$ & 868 & 928 \\
\hline$\%$ & $74.9 \%$ & $77.1 \%$ \\
\hline \multicolumn{3}{|c|}{ Unadjusted percentage-point difference* } \\
\hline $95 \% \mathrm{Cl}$ & Reference & $2.2(-1.2$ to 5.7$)$ \\
\hline$P$ value & & 0.20 \\
\hline \multicolumn{3}{|c|}{ Adjusted percentage-point difference $†$} \\
\hline $95 \% \mathrm{Cl}$ & Reference & $2.5(-0.9$ to 5.9$)$ \\
\hline $\mathrm{P}$ value & & 0.15 \\
\hline
\end{tabular}

*Ordinary least squares regression models without adjustment. †Ordinary least squares regression models adjusted for incentive arm (type, value and typexvalue) as well as participant's age, highest completed education level and marital status.

contrast, among 586 participants who were enrolled $\leq 40$ days before the CHC, the effect of planning prompts on HIV testing uptake was twice as large (3.6 percentagepoint increase in testing due to planning prompts; $95 \%$ CI -2.9 to $10.1, \mathrm{p}=0.27)$. Neither effect was statistically significant. In other post hoc subgroup analyses, the planning prompt intervention did not have a significant effect within each of the incentive arms of the parent trial, nor when examining men tested for HIV within the past year or not (online supplemental appendix table 3).

\section{DISCUSSION}

These findings suggest that behavioural interventions and 'nudges' such as planning prompts have the potential to increase the uptake of HIV testing among men in sub-Saharan Africa, particularly when introduced shortly before testing opportunities. The increases in HIV testing uptake that we found, while not statistically significant at conventional levels, were consistent with large-scale applications of planning prompts for other behaviours. ${ }^{5} 14$ In a prominent study of planning prompts to promote influenza vaccination in the USA, for example, the intervention increased vaccination rates by 4.2 percentage points. ${ }^{5}$ At the population level, an increase in HIV testing of 2-3 percentage points at low or no cost would have a meaningful impact on the percentage of persons living with HIV who know their status. To our knowledge, no previous study has evaluated the application of planning prompts to HIV testing. The encouraging findings from this study suggest that future work should use larger sample sizes to more precisely measure the effect of planning prompts on testing and other health behaviours.

Planning prompts appear to be most effective when applied to discrete, appointment-based interventions or behaviours. ${ }^{12}$ In this way, they could promote a variety of screening behaviours in addition to HIV testing, engagement of HIV-positive individuals in the treatment cascade, or caregivers' timely vaccinations of their children. Our subgroup analyses also suggested that an important feature of planning prompts may be their proximity in time to the planned behaviour. The intervention appeared to be more effective among participants enrolled $\leq 40$ days before the CHC than among those enrolled 41-80 days beforehand, although the effects in both groups were not statistically significant. The importance of its proximity to the CHC is consistent with the study of planning prompts in other contexts, ${ }^{16}$ such as influenza vaccinations, in which the intervention was provided 1-4 weeks prior to clinic days. ${ }^{5}$ Planning a behaviour closer to the intended date may reduce constraints in making a plan and limit derailments from achieving one's goals. ${ }^{15}$

Importantly, planning prompts are inexpensive to implement and can be easily combined with other interventions. In our study, participants would already have received a printed calendar of the CHCs and a brief explanation of the page, so the implementation of the planning prompt required only a modification of the calendar that encouraged participants to select when they would go for HIV testing. Therefore, the intervention added minimal additional cost to the parent trial. While the calendars were given to participants at their homes during enrolment in the study, other delivery methods that are minimally expensive may be possible in various settings. These include the provision of planning prompts along with informational sheets at bars and high-risk venues, through schools, churches and workplaces, as well as by community mobilisers. Other modes of implementing planning prompts, such as mailers, text messages or online schedulers, may also have applicability in some settings and should be tested further. Moreover, our study combined the planning prompts with an incentive-based intervention. ${ }^{9}$ In this way, a planning prompt could complement other strategies that agencies are already using.

While nudges like defaults and micro-incentives have sometimes yielded large changes in behaviour, planning prompts should be viewed as a low-cost or no-cost way to achieve small but nonetheless meaningful changes in behaviour. As a result, future studies that test planning prompts should consider using much larger sample sizes. Our study was underpowered to detect a 2 percentagepoint change in HIV testing uptake, but a similar effect size in a larger study would likely be significant. Considering the low cost of using planning prompts as part of HIV service delivery, an intervention with such an effect would warrant further scale-up. As with many nudges, planning prompts are also well-suited for rapid testing with experimental designs.

Importantly, this study also contributes to ongoing discussion about the effects of nudges when implemented in a pragmatic, scalable manner by 'nudge units' within government agencies or hospital systems. A forthcoming review of 126 randomised controlled trials of 
interventions implemented at scale by two major nudge units in the USA found that the average impact of nudges was 1.4 percentage points, a meaningful and statistically significant effect for low-cost nudges. ${ }^{17}$ This same review found that, largely due to publication bias, randomised trials of nudges published in academic journals had a much larger effect size of 8.7 percentage points. Our results are far more consistent with the effect of nudges that are typically implemented at scale.

This study has several limitations. First, for some participants, the time between the provision of the planning prompts and the occurrence of CHCs in their communities may have been too long to maximise their effectiveness. As our findings suggest, the planning prompts may have generated a larger increase in HIV testing among those who were prompted shortly before the CHC. Second, while the study had over 2000 participants, it was not powered to detect effect sizes smaller than 5 percentage points. Since a very inexpensive intervention that increases HIV testing uptake by $2-3$ percentage points would likely be considered cost-effective, larger studies that test our intervention and evaluate costeffectiveness should be considered. These studies could confirm that planning prompts increase testing for HIV and fine-tune the conditions under which this intervention is most effective, for example, medium of delivery, wording of prompts, timing of delivery and demographic subgroups that are most receptive to prompts.

\section{CONCLUSION}

This study provides suggestive evidence indicating that 'nudges' such as planning prompts should be tested more widely to promote HIV prevention and treatment in sub-Saharan Africa. Financial incentives, defaults and reminders are examples for which insights from behavioural economics have been used to increase the utilisation of HIV services, ${ }^{4-102021}$ and this study expands the set of interventions that should be considered for further study by researchers and programmes. Identifying and evaluating low-cost and no-cost planning prompts and other 'nudges' would be especially valuable to practitioners in settings with fewer resources and a greater burden of HIV.

\footnotetext{
Author affiliations

${ }^{1}$ Department of Medical Ethics and Health Policy, University of Pennsylvania Perelman School of Medicine, Philadelphia, Pennsylvania, USA

${ }^{2}$ Data Science to Patient Value, University of Colorado School of Medicine, Anschutz Medical Campus, Aurora, Colorado, USA

${ }^{3}$ Infectious Diseases Research Collaboration, Kampala, Central Region, Uganda ${ }^{4}$ Division of HIV, Infectious Diseases and Global Medicine, University of California San Francisco, San Francisco, California, USA

${ }^{5}$ Department of Medicine, Makerere University, Kampala, Kampala, Uganda ${ }^{6}$ Center for Health Incentives and Behavioral Economics, University of Pennsylvania Perelman School of Medicine, Philadelphia, Pennsylvania, USA
}

Twitter Nolan M Kavanagh @nolankavanagh

Acknowledgements The authors would like to thank the residents of the study communities for their generous participation in our study as well as research assistants who helped implement the study. This work was supported by a grant
(R01MH105254) from the National Institute of Mental Health (NIMH) at the National Institutes of Health.

Contributors GC, DK and HT designed the study. GC, DK, DH, MRK and HT developed the study protocol. AN, EMS and DK supervised the data collection. AN and KM helped organise data collection and set up the study database. NMK and EMS carried out the statistical analysis. NMK and HT wrote the first draft of the article. All authors reviewed the manuscript.

Funding This study was funded by National Institute of Mental Health (grant number: R01MH105254).

Competing interests None declared.

\section{Patient consent for publication Not required.}

Ethics approval All participants provided written informed consent before participation in the trial. The study protocol was approved by the Makerere University School of Medicine Research and Ethics Committee (in Uganda), the Ugandan National Council on Science and Technology and the University of California San Francisco Committee on Human Research (USA).

Provenance and peer review Not commissioned; externally peer reviewed.

Data availability statement De-identified participant data and protocols are available on request to the corresponding author at hthirumu@pennmedicine. upenn.edu.

Supplemental material This content has been supplied by the author(s). It has not been vetted by BMJ Publishing Group Limited (BMJ) and may not have been peer-reviewed. Any opinions or recommendations discussed are solely those of the author(s) and are not endorsed by BMJ. BMJ disclaims all liability and responsibility arising from any reliance placed on the content. Where the content includes any translated material, BMJ does not warrant the accuracy and reliability of the translations (including but not limited to local regulations, clinical guidelines, terminology, drug names and drug dosages), and is not responsible for any error and/or omissions arising from translation and adaptation or otherwise.

Open access This is an open access article distributed in accordance with the Creative Commons Attribution Non Commercial (CC BY-NC 4.0) license, which permits others to distribute, remix, adapt, build upon this work non-commercially, and license their derivative works on different terms, provided the original work is properly cited, appropriate credit is given, any changes made indicated, and the use is non-commercial. See: http://creativecommons.org/licenses/by-nc/4.0/.

ORCID iD

Nolan M Kavanagh http://orcid.org/0000-0002-5701-0993

\section{REFERENCES}

1 UNAIDS. Fast-Track: ending the AIDS epidemic by 2030. New York City: UNAIDS, 2014. https://www.unaids.org/en/resources/ documents/2014/JC2686_WAD2014report

2 UNAIDS. Unaids data 2019. New York: UNAIDS, 2019.

3 Chamie G, Clark TD, Kabami J, et al. A hybrid mobile approach for population-wide HIV testing in rural East Africa: an observational study. Lancet HIV 2016;3:e111-9.

4 Johnson EJ, Goldstein D. Medicine. do defaults save lives? Science 2003;302:1338-9.

5 Milkman KL, Beshears J, Choi JJ, et al. Using implementation intentions prompts to enhance influenza vaccination rates. Proc Natl Acad Sci U S A 2011;108:10415-20.

6 Banerjee AV, Duflo E, Glennerster R, et al. Improving immunisation coverage in rural India: clustered randomised controlled evaluation of immunisation campaigns with and without incentives. BMJ 2010;340:c2220.

7 Lester RT, Ritvo P, Mills EJ, et al. Effects of a mobile phone short message service on antiretroviral treatment adherence in Kenya (WelTel Kenya1): a randomised trial. Lancet 2010;376:1838-45.

8 Pop-Eleches C, Thirumurthy H, Habyarimana JP, et al. Mobile phone technologies improve adherence to antiretroviral treatment in a resource-limited setting: a randomized controlled trial of text message reminders. AIDS 2011;25:825-34.

9 Chamie G, Schaffer EM, Ndyabakira A, et al. Comparative effectiveness of novel nonmonetary incentives to promote HIV testing. AIDS 2018;32:1443-51.

10 Montoy JCC, Dow WH, Kaplan BC. Patient choice in opt-in, active choice, and opt-out HIV screening: randomized clinical trial. BMJ 2016;532:h6895. 
11 Thaler RH, Sunstein CR. Nudge: improving decisions about health, wealth, and happiness. 1 edn. New Haven: Yale University Press, 2008.

12 Gollwitzer PM. Implementation intentions: strong effects of simple plans. Am Psychol 1999;54:493-503.

13 Sheeran P, Orbell S. Using implementation intentions to increase attendance for cervical cancer screening. Health Psychol 2000;19:283-9.

14 Conner M, Higgins AR. Long-Term effects of implementation intentions on prevention of smoking uptake among adolescents: a cluster randomized controlled trial. Health Psychol 2010;29:529-38.

15 Gollwitzer PM, Sheeran P. Implementation Intentions and Goal Achievement: A Meta-analysis of Effects and Processes. In: Advances in experimental social psychology. Academic Press, 2006: 69-119.
16 Sheeran P, Milne S, Webb TL, et al. Implementation Intentions and Health Behaviour. In: Predicting health behavior. New York: Open University Press, 2005: 276-323.

17 DellaVigna S, Linos E. Rcts to scale: comprehensive evidence from two Nudge units. University of California Berkeley, 2020.

18 Uganda Ministry of Health. Uganda national policy guidelines for HIV voluntary counselling and testing. Kampala: Ministry of Health, 2005.

19 Wooldridge JM. Econometric Analysis of Cross Section and Panel Data. second edition edition. Cambridge, Mass: The MIT Press, 2010.

20 Galárraga O, Sosa-Rubí SG. Conditional economic incentives to improve HIV prevention and treatment in low-income and middleincome countries. Lancet HIV 2019;6:e705-14.

21 Thirumurthy $\mathrm{H}$, Masters SH, Rao S, et al. Effect of providing conditional economic compensation on uptake of voluntary medical male circumcision in Kenya: a randomized clinical trial. JAMA 2014;312:703-11. 